\title{
Correction To: Application of Quasi-Steady-State Methods to Nonlinear Models of Intracellular Transport by Molecular Motors
}

Cole Zmurchok ${ }^{1}$ (D) - Tim Small ${ }^{1}$.

Michael J. Ward $^{1}$ - Leah Edelstein-Keshet ${ }^{1}$

\section{Correction To: Bull Math Biol (2017) 79:1923-1978 https://doi.org/10.1007/s11538-017-0314-1}

We apologize for the error in the references. The correct citation is:

Yochelis A., Bar-On T., Gov N.S. (2016) Reaction-diffusion-advection approach to spatially localized treadmilling aggregates of molecular motors. Physica D: Nonlinear Phenomena 318-319:84-90.

The original article can be found online at https://doi.org/10.1007/s11538-017-0314-1.

$\triangle$ Cole Zmurchok

zmurchok@math.ubc.ca

1 Department of Mathematics, University of British Columbia, Vancouver V6T 1Z2, Canada 